\title{
INFLUENCE OF NON-LINEAR WAVES AND UNDERTOW IN SANDBAR DEVELOPMENT
}

\author{
Tiago Abreu' ${ }^{1}$ Francisco Sancho ${ }^{2}$ and Paulo A. Silva ${ }^{3}$
}

\begin{abstract}
As waves propagate from deep into shallow waters, they begin to interact with the sea floor and undergo through several changes due to non-linear effects. These interactions cause the waves to transform and become non-linear as they decelerate and finally break. These local nonlinearities are reflected on the near-bed oscillatory flow and are inextricably linked to sediment transport, causing erosion-accretion patterns and bar migration. In this work the ability of a practical transport model that includes the effects of velocity and acceleration skewness in the timevarying bed shear stress (Abreu et al., 2011) is assessed to predict sediment transport rates under oscillatory flows and currents. The results are compared with two different data sets obtained under sheet flow conditions, showing a good agreement with the measurements. Moreover, its performance to the undertow and to non-linear characteristics is further assessed. The practical sediment transport model is coupled to a simple bed-evolution model, enabling to evidence the relative strength of mechanisms associated with the wave and current induced sand transports. The results show that the formation of the bar and its migration is affected by a new term introduced in the bed shear stress predictor. This time-varying term accounts for the shape of the wave and is described through two non-linear parameters recently proposed in Abreu et al. (2010). This work provides further insights in the correct prediction of sediment transport modeling and sandbar developments, due to the combined influence of non-linear waves with undertow currents.
\end{abstract}

Keywords: non-linear waves; skewness; asymmetry; undertow; sandbar

\section{INTRODUCTION}

Studies on sediment transport are of paramount importance in marine coastal environments. The movement of sediments is mainly caused by the action of waves or currents, or a combination of both. For increasingly shallow waters, these hydrodynamic processes induce motions on the seabed and if the grains are exposed to large enough velocities they start to move. The interplay between sediment transport and water motion is also affected by non-linear interactions owing to propagating waves. As ocean surface waves approach the coast and propagate into shallower water they transform in appearance, becoming asymmetric and skewed. These local nonlinearities are reflected on the near-bed oscillatory flow and are inextricably linked to sediment transport. For example, the asymmetry of the wave leads to acceleration-skewed orbital motions, resulting in non-zero net sediment transport as shown by recent experimental studies performed in full-scale laboratory experiments (e.g., van der A et al., 2010, Silva et al., 2011).

In this work the effects of the wave hydrodynamics interacting with the sea floor are analyzed in the direction of wave propagation, resulting into a two-dimensional analysis (2D). Since, often, the incoming waves become almost perpendicular to the nearshore bathymetry, this study can be considered to contribute to the cross-shore transport problem.

This work intends to capture the relative strengths of the main hydrodynamic processes driving onshore and off-shore transport involved in the cross-shore direction. Whereas the near-bottom offshore directed mean flows (undertow currents) play a crucial role in transporting sediment in the offshore direction, the nonlinearities associated to the wave motion can drive sediment transport in the onshore direction. This has several implications for profile developments, causing the existence of a convergence of sediment at certain locations, generating sandbars or contributing to sandbar migration patterns.

To characterize orbital motions under waves with skewness and asymmetry, the present paper considers a simple analytical free-stream velocity description presented by Abreu et al. (2010). The formula contains four free parameters: two related to the orbital velocity amplitude and wave period and two related to the velocity and acceleration skewnesses, namely, an index of skewness, $r$, and a waveform parameter, $\phi$. Moreover, Abreu et al. (2011a) incorporated the parameters $r$ and $\phi$ in a new instantaneous bed shear stress parameterization for arbitrary non-linear, skewed and asymmetric,

${ }^{1}$ Civil Engineering Department \& CESAM, Polytechnic Institute of Viseu, ESTGV Campus de Repeses, Viseu, 3504510, Portugal, tabreu@estv.ipv.pt

2 Hydraulics and Environment Department, National Laboratory for Civil Engineering, Av. do Brasil, 101, Lisboa, 1700-066, Portugal, fsancho@lnec.pt

${ }^{3}$ Physics Department \& CESAM, University of Aveiro, Campus Universitário de Santiago, Aveiro, 3810-193,

Portugal, psilva@ua.pt 
oscillatory flows. The performance to predict sediment transport rates when incorporated in the quasisteady bed load formulation of Nielsen (2006) is assessed using two different data sets under sheet flow conditions (van der A et al., 2010, and Silva et al., 2011). Moreover, its ability to predict transport rates under non-linear characteristics and mean currents is further assessed.

Recently, Ruessink et al. (2012) proposed parameterizations to estimate $r$ and $\phi$ from the significant wave height, wave period and water depth, enabling its easy introduction in many numerical simulation models for predicting beach profile changes. In this work, the aforementioned parameterizations and the practical sediment transport model are coupled to a simple bed-evolution model, enabling to predict the formation and migration of sandbars. The results are also compared using Nielsen's (2006) original method in order to evidence the differences obtained with the introduction of the new bed shear stress estimator.

\section{NEARSHORE HYDRODYNAMICS}

\section{Wave propagation}

The modeling of surface gravity waves and, particularly, the determination of the wave height, $H$, at arbitrary points is essential in order to compute sediment transport rates and morphological changes. Here, one assumes a simplification of the wave propagation phenomena, by considering the propagation of regular waves perpendicular to the shoreline determined only by wave shoaling and shallow-water wave breaking. Though the consideration of these intervening processes is quite simple, this approach is well suited to provide insight into the physical mechanisms responsible for morphological changes.

In order to account for the wave shoaling, the linear model represented through Eq. (1) and (2) is adopted:

$$
\begin{gathered}
H=K_{s h} H_{0}, \\
K_{s h}=\frac{\cosh k h}{\sqrt{k h+\operatorname{senh} k h \cosh k h}},
\end{gathered}
$$

where $H$ is the wave height, the subscript 0 refers to a position offshore, $K_{\text {sh }}$ represents the shoaling factor, and $k$ is the wave number $(k=2 \pi / L)$.

For the determination of the wavelenght, $L$, at an arbitrary depth $h$, the linear wave theory was used by solving the dispersion relation, $L=L_{0} \tanh (k h)$, where $L_{0}=g T^{2} /(2 \pi)$ is the deep water wave lenght and $g$ is the acceleration due to gravity.

Eq. (1) and (2) are used until the water depth at breaking, $h_{\mathrm{b}}$, is attained. This depth is obtained when the breaker index, i.e., the ratio of the height of a wave to the depth of the water it is breaking into $(H / h)$ equals a maximum of 0.78 (McCowan, 1984). The adoption of this criterion in shallower depths avoids the consideration of exaggerated and unrealistic values of $H$ closest to the shore.

\section{Near-bed orbital velocities}

Wave motion is often thought of as pure sinusoidal movement. This simplest representation of ocean water waves corresponds to the solution of the most elementary wave theory developed by Airy (1841) and it is known as small-amplitude or linear wave theory. According to this theory, the amplitude of the orbital velocity near the bottom, $U_{\mathrm{w}}$, associated to monochromatic waves is given by:

$$
U_{w}=\frac{\pi H}{T \sinh (k h)}
$$

Linear theory provides a most useful first approximation to the wave motion and can be used in many coastal engineering applications due to its simplicity. Nonetheless, ocean waves are generally not "small in amplitude" and, consequently, the theory presents some relevant restrictions concerning sediment transport.

Stokes (1847) was one of the first to note and analyze the nonlinearities prior to wave breaking. He observed that, as waves approach the coast and propagate into shallower water, the waves present shorter and higher crests and longer and shallower troughs than in the sinusoidal profile. Apart from becoming peaky with sharp wave crests and long wave troughs, once waves approach breaking and enter the surf zone, they also attain a sawtooth shape, with a steep front face and more gently sloping 
rear face (e.g., Svendsen et al., 1978). The transformations of the surface elevation are illustrated in Fig. 1 and are usually designated as skewed and asymmetric waves (e.g., Elgar and Guza, 1985).

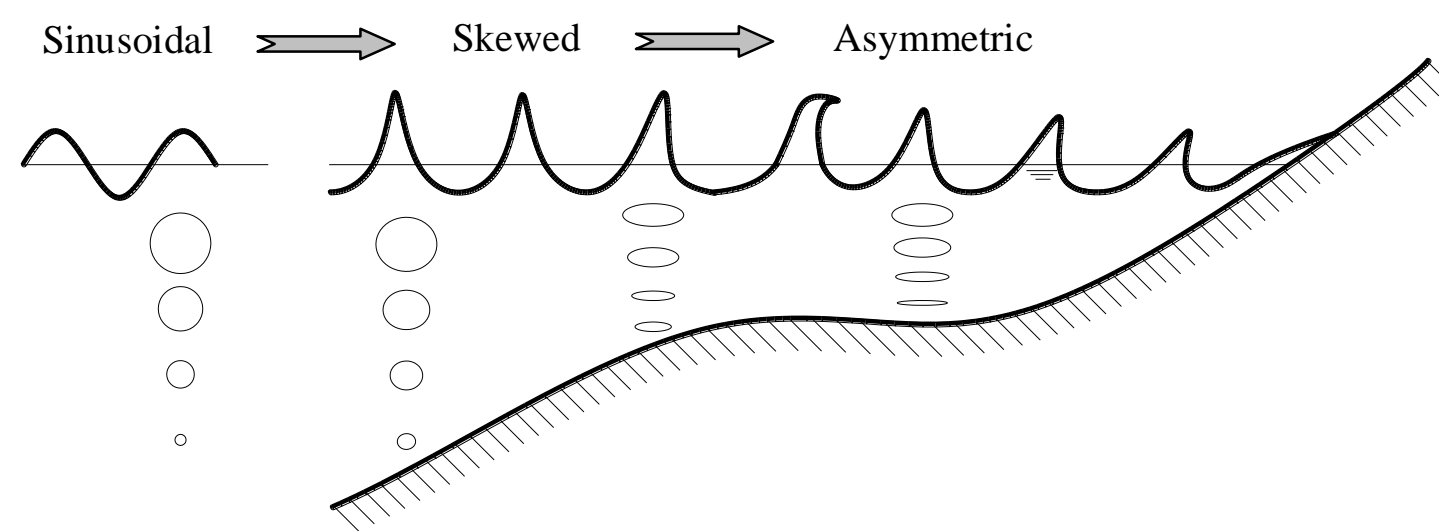

Circular orbits

Elliptical orbits

Figure 1. Sketch of wave shape transformation and corresponding orbital motions as waves propagate from deep to shallow waters.

Moreover, the corresponding orbital velocity near the bottom shows a similar (time) variation. Under the steep front face the velocity varies rapidly from a maximum negative (offshore) value to a maximum positive (onshore) value, giving rise to rapid fluid accelerations. The accelerations induced by the motion of the rear face of the wave are much smaller. This effect is related with the acceleration skewness. Therefore, to characterize orbital motions under waves with skewness and asymmetry, the present paper considers a simple analytical free-stream velocity description presented by Abreu et al. (2010). The formula contains four free parameters: two related to the orbital velocity amplitude and wave period and two related to the velocity and acceleration skewnesses, namely, an index of skewness, $r$, and a waveform parameter, $\phi$ :

$$
u(t)=U_{w} f \frac{\sin (\omega t)+\frac{r \sin \phi}{1+f}}{1-r \cos (\omega t+\phi)}
$$

where $f=\sqrt{1-r^{2}}$.

A purely acceleration-skewed flow (i.e. sawtooth wave) is obtained for $\phi=0$ and a pure velocityskewed flow for $\phi=-\pi / 2$. Between these two extreme values the orbital flow contains both velocity and acceleration skewness as usually found in nature (e.g., Doering and Bowen, 1995; Elfrink et al., 2006; Ruessink et al., 2009).

Fig. 2 illustrates the application of Eq. (4) showing the time varying orbital velocity and the corresponding acceleration for $\phi=-\pi / 4$, representative of a case where both velocity and acceleration skewness coexist. Three values of $r$ were considered: $0,0.4$ and 0.8 . It is possible to deduce that, independently of $\phi$ values, Eq. (4) matches to the sinusoidal wave for $r=0$. However, when $r$ increases, the non-linear behavior is evidenced.

Since the sediment transport computations require a good description of the orbital motion, it is important to link the nonlinearities $r$ and $\phi$ with standard output of nearshore hydrodynamic models as the significant wave height, wave period and water depth. In the literature, it is possible to find several publications that provide it as, for example, in Dibajnia et al. (2001), Elfrink et al. (2006) or Ruessink et al. (2012). All these works enable the practical application of this formulation. In this work we adopt the recent parameterizations proposed by Ruessink et al. (2012) that are based on a data set of more than 30,000 field observations. 

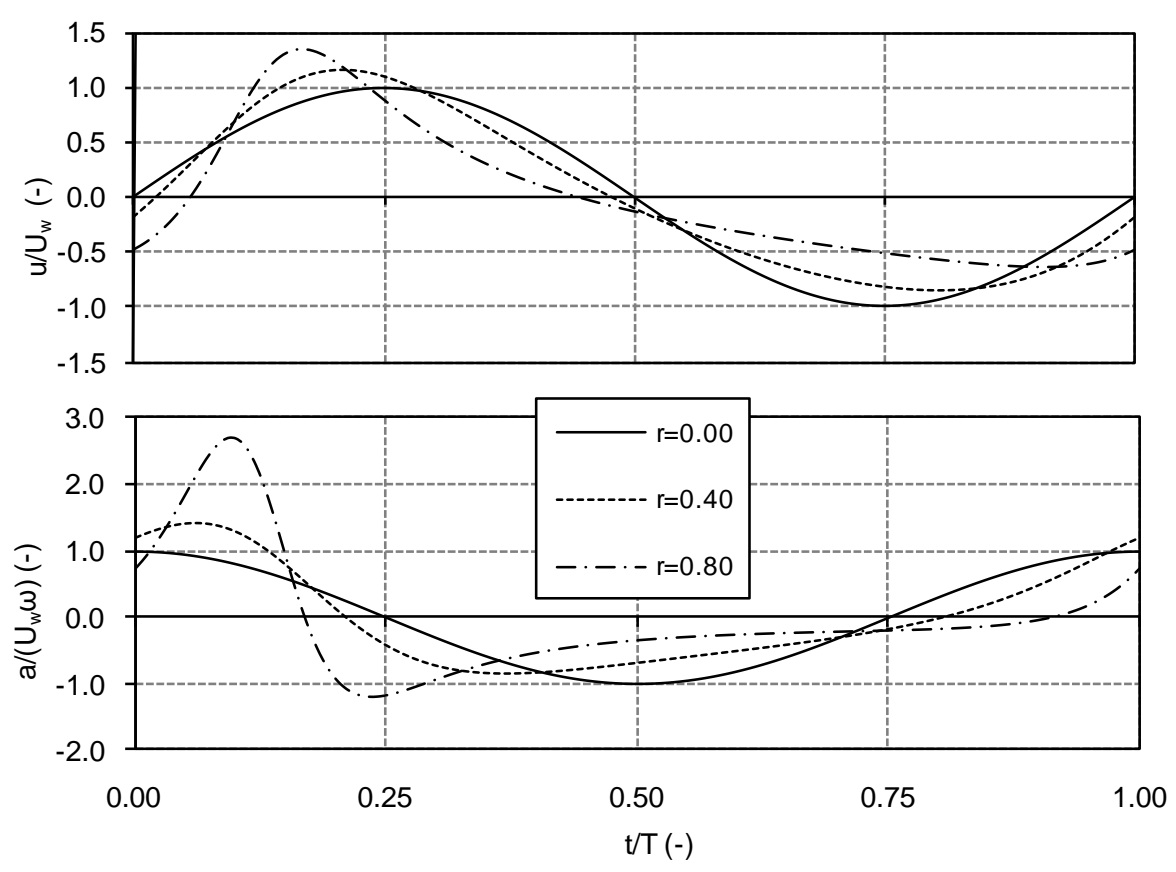

Figure 2. Time varying orbital velocity and acceleration considering $\phi=-\pi / 4$ and $r=0,0.4$ and 0.8 .

These authors provide expressions to obtain $r$ and $\phi$ as function of the Ursell number and a dimensionaless non-linearity parameter $B$ :

$$
\begin{gathered}
U_{r}=\frac{3}{8} \frac{H k}{(k h)^{3}}, \\
B=p_{1}+\frac{p_{2}-p_{1}}{1+\exp \left(\frac{p_{3}-\log U_{r}}{p_{4}}\right)},
\end{gathered}
$$

with $p_{1}=0, p_{2}=0.857, p_{3}=-0.471$ and $p_{4}=0.297$.

The parameterizations to compute $r$ and $\phi$ follow

$$
\begin{gathered}
\phi=-\frac{\pi}{2} \tanh \left(p_{5} / U_{r}^{p_{6}}\right), \\
B=3 b / \sqrt{2\left(1-b^{2}\right)},
\end{gathered}
$$

with $p_{5}=0.815$ and $p_{6}=0.672$ and, after Malarkey and Davies (2012), $b=r /\left(1+\sqrt{1-r^{2}}\right)$.

Instead of solving Eq. (8), one considers the following explicit approximation (Ruessink, personal communication):

$$
r=\tanh (0.931 B)
$$

\section{Undertow model}

In addition to near-bed wave orbital velocities, the sediment transport is also driven by the undertow current at the bottom, $U_{\mathrm{b}}$. This offshore directed current is the result of a mass-flux compensation, since it balances the mass transported onshore by breaking waves within the surf zone.

To calculate the undertow current the explicit model developed by Rattanapitikon and Shibayama (2000) is chosen. Their model was validated with a wide range of laboratory data covering small and 
large-scale experiments, providing good estimations of $U_{\mathrm{b}}$. The surf zone is divided into a transition zone and an inner zone and the undertow in these two zones is discussed separately:

$$
U_{b}=-0.76 \frac{B_{0} g H^{2} T}{h L}-1.12 b_{3} \frac{B_{0} L H}{h T},
$$

with

$$
b_{3}=\left\{\begin{array}{cc}
0 & \text { offshore zone } \\
\frac{1 / H-1 / \sqrt{H_{b}}}{1 / H_{t}-1 / \sqrt{H_{b}}} & \text { transition zone } \\
1 & \text { inner surf zone }
\end{array}\right.
$$

and

$$
B_{0}=0.125+0.6 m-0.089 \frac{H}{h},
$$

where $H_{\mathrm{b}}$ and $H_{\mathrm{t}}$ represent the wave heights at the breaking and transition cross-shore positions and $m$ is the mean bottom slope. Here, the transition point has been assumed a certain distance $(5 \mathrm{~m}$ for the present applications) shoreward the breaking point.

\section{SEDIMENT TRANSPORT}

\section{Practical sediment transport model}

The recognition of the importance of wave asymmetry to nearshore sediment transport has motivated the development of practical sand transport models including this process (e.g., Drake and Calantoni, 2001; Hoefel and Elgar, 2003; Nielsen, 2006; Silva et al., 2006) and numerical studies with detailed bottom boundary layer models (e.g., Drake and Calantoni, 2001; Ruessink et al., 2009).

The practical models are extensions of well-known previous formulations (e.g., Meyer-Peter and Müller, 1948) and describe the effect of wave asymmetry in sediment transport by either considering an increase of bed shear stress induced by the large flow accelerations, as suggested by Nielsen (1992), or through the inclusion of the free stream acceleration directly. The more sophisticated process-based models allow examining in more detail the physical mechanisms underlying the effect of wave shape on sediment transport but do not necessary lead to better sediment transport rate estimates in comparison with relatively simple practical models (Davies et al., 2002).

To predict the spatial distribution of the net sediment transport rates, one has taken into account a new bed shear stress estimator (Abreu et al., 2011a). This new predictor extends the works of Nielsen (1992, 2002), Nielsen and Callaghan (2003) and Terrile et al. (2009) and considers the same parameters $(r$ and $\phi$ ) proposed for the fluid velocity description of Eq. (4).

The corresponding time-varying bed shear stress near the bottom follows:

$$
\tau(t)=\rho u_{*}(t)\left|u_{*}(t)\right|
$$

with the shear velocity $u_{*}$ defined as

$$
u_{*}(t)=\sqrt{\frac{f_{w}}{2}}\left(\cos (\varphi) u(t)+\frac{\sin (\varphi)}{\omega}\left[\frac{d u(t)}{d t}-S(t, \phi, r)\right]\right)
$$

where $\omega$ is the angular frequency, $f_{\mathrm{w}}$ the wave friction factor and, similar to Nielsen, the angle $\varphi$ is a calibrating parameter that, in the case of a single harmonic, roughly represents the phase lead of bed shear stress over the free stream velocity. The parameter $\varphi$ establishes the balance between drag forces and pressure gradients associated with the cosine and sine of $\varphi \in\left[0^{\circ}, 90^{\circ}\right]$, respectively.

It is noticed that the inclusion of the new term $S(t, \phi, r)$ corrects the acceleration term and introduces the shape of the wave described through $r$ and $\phi$, bringing in more physics. The expression of $S$ yields 


$$
S(t, \phi, r)=\omega \cdot f \cdot U_{w} \frac{r[-(-1+f) \cos \phi-2 r \cos (\omega t)+(1+f) \cos (2 \omega t+\phi)]}{2(1+f)[-1+r \cos (\omega t+\phi)]^{2}} .
$$

To compute the wave friction factor $f_{\mathrm{w}}$ in Eq. (14) we use Nielsen's (1992) formulation

$$
f_{w}=\exp \left[5.5\left(\frac{k_{s}}{A_{r m s}}\right)^{0.2}-6.3\right]
$$

where $k_{\mathrm{s}}$ represents the bed roughness and $A_{\mathrm{rms}}$ is the representative near bed semi-excursion.

To estimate the sediment transport rates, qs, the new bed shear stress predictor is incorporated in Nielsen's (2006) bedload formula, which is a modified version of the Meyer-Peter Müller (1948) bedload type formula:

$$
q_{s}=12 \sqrt{(s-1) g d^{3}}\left(\theta(t)-\theta_{c r}\right) \sqrt{\theta(t)} \frac{u_{*}}{\left|u_{*}\right|}, \theta>\theta_{c r}
$$

where the instantaneous Shields parameter $\theta(t)$ is defined by $\theta(t)=u_{*}{ }^{2}(t) /\left((s-1) g d_{50}\right), s=\rho_{\mathrm{s}} / \rho$ is the ratio between sediment and water densities, $d_{50}$ is the median grain size, $\theta_{\mathrm{cr}}$ is the critical value of $\theta$ at the threshold of motion. A typical value of $\theta_{\mathrm{cr}}=0.05$ is assumed for the present conditions.

Abreu et al. (2011a) tested this new methodology against the measured sediment transport rates of van der A et al. (2010) and, using the default value $\varphi=51^{\circ}$ recommended by Nielsen (2006), noted that the estimated transport rates presented better predictive trends compared no Nielsen's original method. The experiments of van der A et al. (2010) were obtained at the Aberdeen Oscillatory Flow Tunnel and involved acceleration-skewed flows under sheet flow conditions, considering three different median grain sizes $\left(d_{50}=0.15,0.27\right.$ and $\left.0.46 \mathrm{~mm}\right)$. These oscillatory flows also contained a small degree of velocity skewness, which was taken into account in the new formulation through $r$ and $\phi$. However, when using $k_{\mathrm{s}}=2.5 d_{50}$ to compute the wave friction factor, Abreu et al. (2011a) found some disagreement in the magnitude of the transport rates: the medium and coarse sands are generally within a factor of about 2 , while the fine sands are under-estimated by a higher factor.

Abreu (2011) remarked that much better agreement between predicted and measured transport rates can be obtained if $f_{\mathrm{w}}$ is calibrated separately for the different sand sizes. For example, Fig. 3 shows that using $k_{\mathrm{s}}=25 d_{50}, 15 d_{50}, 2.5 d_{50}$ and $d_{50}$, for $d_{50}=0.15,0.20,0.27$ and $0.46 \mathrm{~mm}$, respectively, most of the results lie over the line of perfect agreement. The figure considers two different data sets: the one obtained at the Aberdeen Oscillatory Flow Tunnel (van der A et al., 2010) and another at the Large Oscillating Water Tunnel of Deltares performed with a median grain diameter of $d_{50}=0.20 \mathrm{~mm}$ (Silva et al., 2011). It is shown that the use of the new estimator leads into accurate estimations of the net sediment transport rates, approaching the line of perfect agreement. Essentially this means that a smaller grain size implies a proportionally larger roughness and, consequently, friction factor. This can translate that the bed roughness is approximately equal to the grain size for medium sands, but for fine sands it has to be enhanced in relation to sand size, in order to account for the sediment transport component. 


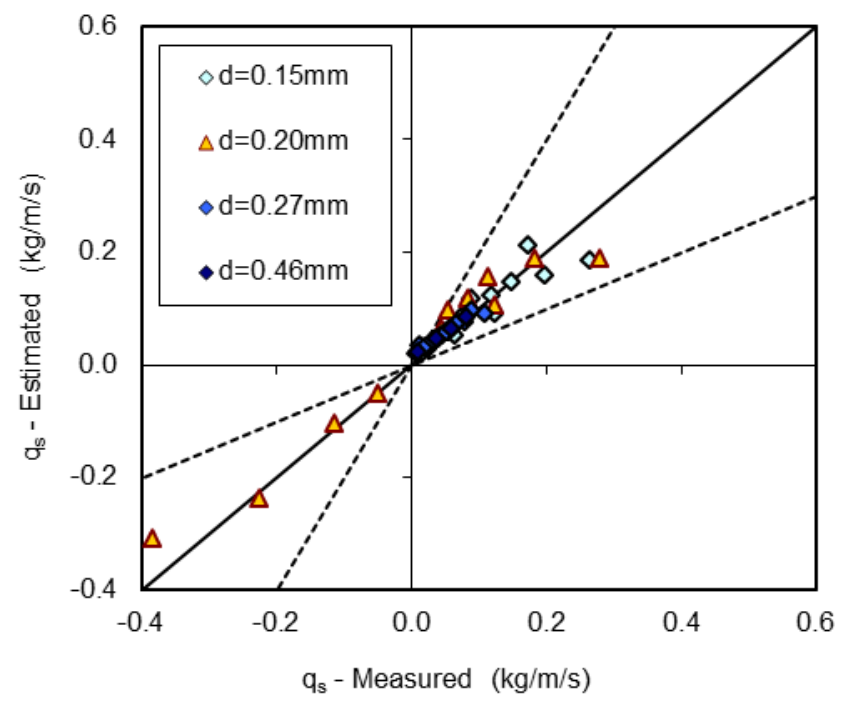

Figure 3. van der A et al. (2010) and Silva et al. (2011) measured net transport rates versus predicted with the new method to compute bed shear stress, using $\varphi=51^{\circ}$ and $k_{\mathrm{s}}=25 d_{50}, 15 d_{50}, 2.5 d_{50}$ and $d_{50}$ for $d_{50}=0.15$, $0.20,0.27$ and $0.46 \mathrm{~mm}$, respectively.

\section{Sensitivity to the undertow and to the non-linear characteristics}

At present, there are very few laboratory experiments, enabling the evaluation of the combined effect of non-linear oscillatory flow motions with opposing net currents, as the undertow. Fig. 3 contemplates four hydrodynamic conditions performed by Silva et al. (2011) that consider simultaneously oscillatory acceleration-skewed flows with opposing net currents $\left(U_{\mathrm{b}}\right)$. These test conditions possess the same flow period $T=7 \mathrm{~s}$ and present negative transport rates. They concern sawtooth shapes (i.e., $\phi=0)$ and were obtained using different values of $r(\approx 0.28$ and 0.44$)$ which reflect different degrees of acceleration skewness.

Fig. 4 plots Silva et al. (2011) net transport rates, $q_{\mathrm{s}}$, measurements as function of $U_{\mathrm{b}}$. The results for $U_{\mathrm{b}}<0$ have a net negative transport rate, i.e. against the direction of maximum acceleration, and an increase in acceleration skewness reduces the amount of sediment transported in the negative direction. Furthermore, Fig. 4 suggests that there is a balance between these nonlinearities and the current, corresponding to a reversal of the sediment transport direction. However, one notes that the range of the experimental test conditions in terms of $r, \phi$ and, particularly, $U_{\mathrm{b}}$ is rather limited.

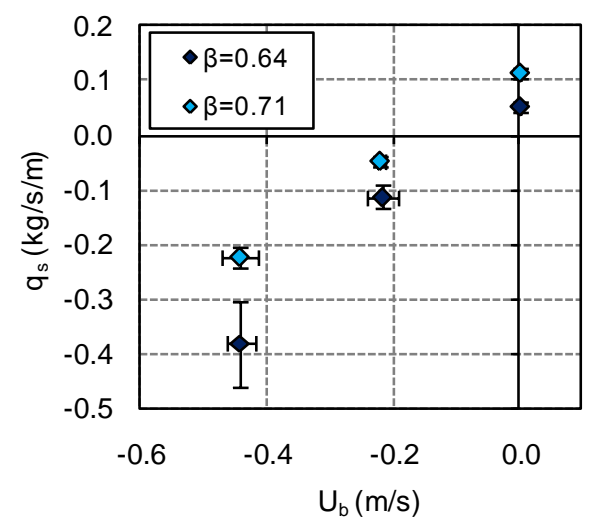

Figure 4. Measured $q_{\mathrm{s}}$ versus $U_{\mathrm{b}}$, considering $r \approx 0.28$ and 0.44 (Silva et al., 2011). The error bars correspond to the standard deviation. 
The performance of the new bed shear stress predictor to estimate measured transport rates is explored and addressed in Fig. 5. The figure plots the measured values of $q_{\mathrm{s}}$ as function of $U_{\mathrm{b}}$ and provides estimated values of $q_{\mathrm{s}}$ using the new predictor for different values of $r$. The hydrodynamic conditions can be considered similar to those of Silva et al. (2011), since the results are obtained with $T=7 \mathrm{~s}, U_{\mathrm{w}}=1.2 \mathrm{~m} / \mathrm{s}, \mathrm{d}_{50}=0.20 \mathrm{~mm}$. The analysis reflects two values of $\phi(0$ and $-\pi / 4)$ and four of $r$ $(0.2,0.4,0.6$ and 0.8$)$, using $\varphi=51^{\circ}$ and $k_{\mathrm{s}}=15 d_{50}$. On the left panel, pure accelerated skewed flows are considered $(\phi=0)$ and on the right panel $(\phi=-\pi / 4)$ the combined effect of acceleration and velocity skewness are presented. As can be seen, the estimates produce positive transport rates for $U_{\mathrm{b}}=0$, which reduce to 0 at a threshold value of $U_{\mathrm{b}}$, and then become negative as $\left|U_{\mathrm{b}}\right|$ increases further. The estimated results corroborate the experimental trends. Also, with a change from asymmetric $(\phi=0)$ to skewedasymmetric $(\phi=-\pi / 4)$ oscillatory flow, the absolute value of the threshold $U_{\mathrm{b}}$ corresponding to $q_{\mathrm{s}}=0$ increases with $r$.
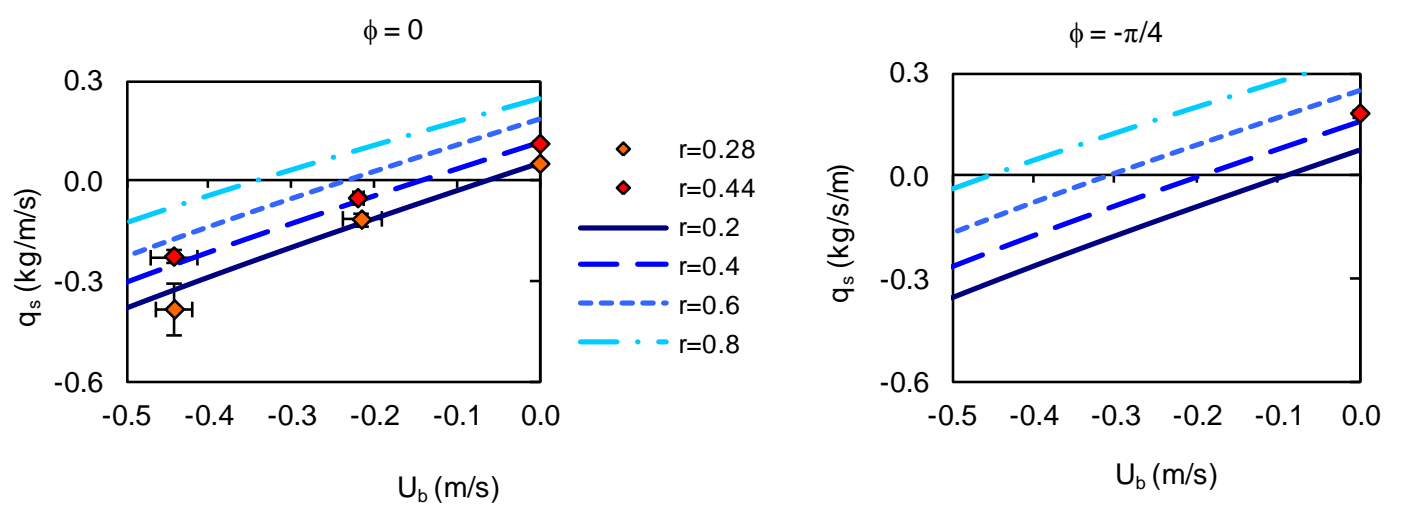

Figure 5. Measured (diamonds) and estimated (lines) transport rates versus $U_{\mathrm{b}}$. The simulations are based on $T=7 \mathrm{~s}, U_{\mathrm{w}}=1.2 \mathrm{~m} / \mathrm{s}, d_{50}=0.20 \mathrm{~mm}, \phi=0$ and $-\pi / 4$ with $r=0.2,0.4,0.6$ e 0.8 .

\section{MORPHODYNAMIC MODEL}

After the sediment transport rates are determined by the sediment transport formula, the morphological changes can be obtained through the mass conservation equation:

$$
\frac{d z_{b}}{d t}=\frac{1}{1-\varepsilon_{0}} \frac{d q_{s}}{d x}
$$

where $z_{b}$ is the bed elevation, $t$ the time, $x$ the horizontal coordinate and $\varepsilon_{0}$ the sand porosity. Following Thornton et al. (1996), one assumes $\varepsilon_{0}=0.3$.

In practice, Eq. (18) needs to be solved numerically. In the literature, numerous finite different schemes can be found for such purpose. In this work, for simplicity, the two-step Lax-Wendroff FTCS (forward time, central space) scheme proposed by Richtmyer (1962) was adopted. This is a second order central difference scheme that requires the calculation of sediment transport rate at intermediate time levels. The Richtmyer scheme is written as:

$$
\begin{gathered}
z_{b, i}^{k+1 / 2}=\frac{z_{b, i+1}^{k}+z_{b, i}{ }^{k}}{2}-\frac{\Delta t}{2 \Delta x} \frac{1}{\left(1-\varepsilon_{0}\right)}\left[\hat{q}_{s, i+1}^{k}-\hat{q}_{s, i}^{k}\right], \\
z_{b, i}^{k+1}=z_{b, i}^{k}-\frac{\Delta t}{\left(1-\varepsilon_{0}\right) \Delta x}\left[\hat{q}_{s, i}^{k+1 / 2}-\hat{q}_{s, i-1}^{k+1 / 2}\right],
\end{gathered}
$$

where $\Delta x$ is the grid spacing, $\Delta t$ the time step, $z_{b, i}{ }^{k+1}$ the updated bed elevation at time $k+1$ found from the sediment transport rate and the bed elevation at time $k$. The values of $\hat{q}_{s}$ are approximations for the 
sediment transport rates $q_{\mathrm{s}}$ obtained using a simple linear smoothing operator of $4^{\text {th }}$ order constructed for discretized functions (Shapiro, 1975):

$$
\hat{q}_{s, i}=\frac{1}{256}\left(-q_{s, i-4}+8 q_{s, i-3}-28 q_{s, i-2}+56 q_{s, i-1}+186 q_{s, i}+56 q_{s, i+1}-28 q_{s, i+2}+8 q_{s, i+3}-q_{s, i+4}\right) .
$$

In addition to the Shapiro smoothing and as suggested by De Vriend et al. (1993), a downwardsloping avalanching term was also included to compute the sediment transport rate $q_{\mathrm{s}}$

$$
\hat{q}_{s, i}=\hat{q}_{s, i}-\varepsilon_{s}\left|\hat{q}_{s, i}\right| \frac{d h}{d x},
$$

where $\varepsilon_{\mathrm{s}}=2$ was adopted.

For the boundary conditions, i.e., at the water depths at most seaward $(i=1)$ and shoreward boundaries $(i=e n d)$, one imposed $h_{1}^{k+1}=h_{1}^{k+1 / 2}$ and $h_{\text {end }}^{k+1 / 2}=h_{\text {end }}^{k}$.

\section{APPLICATION AND RESULTS}

In order to evaluate the performance of the bed shear stress and sediment transport models, to predict beach profile evolutions, an initial "equilibrium" cross-shore profile as proposed by Bruun (1954) is considered. The cross-shore profile increases exponentially with the distance $x$ from the shoreline according to the equation:

$$
h=A x^{m}
$$

where $h$ represents the water depth, $A$ is a sediment scale parameter and $m$ is a dimensionless exponent.

Dean (1991) examined the forms of equilibrium beach profiles in the surf zone and, by fixing an average value for $m$ equal to 2/3, an empirical correlation between $A$ and the mean grain size $d_{50}$ was established:

$$
A=0.21 d_{50}^{0.48}
$$

While recognizing the practical importance of Eq. (23) for preliminary design considerations as, for example, in beach nourishments, the concept of equilibrium profiles is a rather crude representation of beach profiles since it neither includes nor explains the occurrence of bar formations. This work simulates numerically the beach deformation for a period equal to $24 \mathrm{~h}$, starting from a Bruun/Dean "equilibrium" cross-shore profile obtained with $d_{50}=0.20 \mathrm{~mm}$. The sediment transport model only takes into account the wave-induced and undertow-induced transports.

The numerical simulations consider regular waves with $T=7 \mathrm{~s}$ and $H_{0}=1.5 \mathrm{~m}$. Fig. 6 shows the spatial variation of $H, U_{\mathrm{w}}, U_{\mathrm{b}}, r, \phi$ and of the mobility number $\left(=u_{\max }^{2} /(s-1) g d_{50}\right)$ along $600 \mathrm{~m}$, evidencing the initial conditions prior to morphologic changes. The beach profile considered is plotted in the lower panel. The mobility number indicates that sheet flow conditions are achieved since the values are generally greater than 300 (O’Donoghue et al., 2006). This illustrates that the hydrodynamic conditions are close to those of Silva et al. (2011) and therefore, according to Fig. 3, the practical model reasonably captures the relative strength of mechanisms associated with the waves and currents.

For decreasing depths, Fig. 6 shows that the values of $H$ arise continuously until $1.7 \mathrm{~m}$ due to the shoaling process. At such position $\left(x_{\mathrm{b}}=107.0 \mathrm{~m}\right)$ the waves break and, for shallower depths, the values of $H$ reduce in line with the adopted breaking criterion. Similar cross-shore variations are obtained for the orbital velocity near the bottom, which is directly linked to $H$. As expected, it is observed that the values of $U_{\mathrm{b}}$ are negative and its magnitude particularly relevant in the inner surf zone where larger values are attained. 

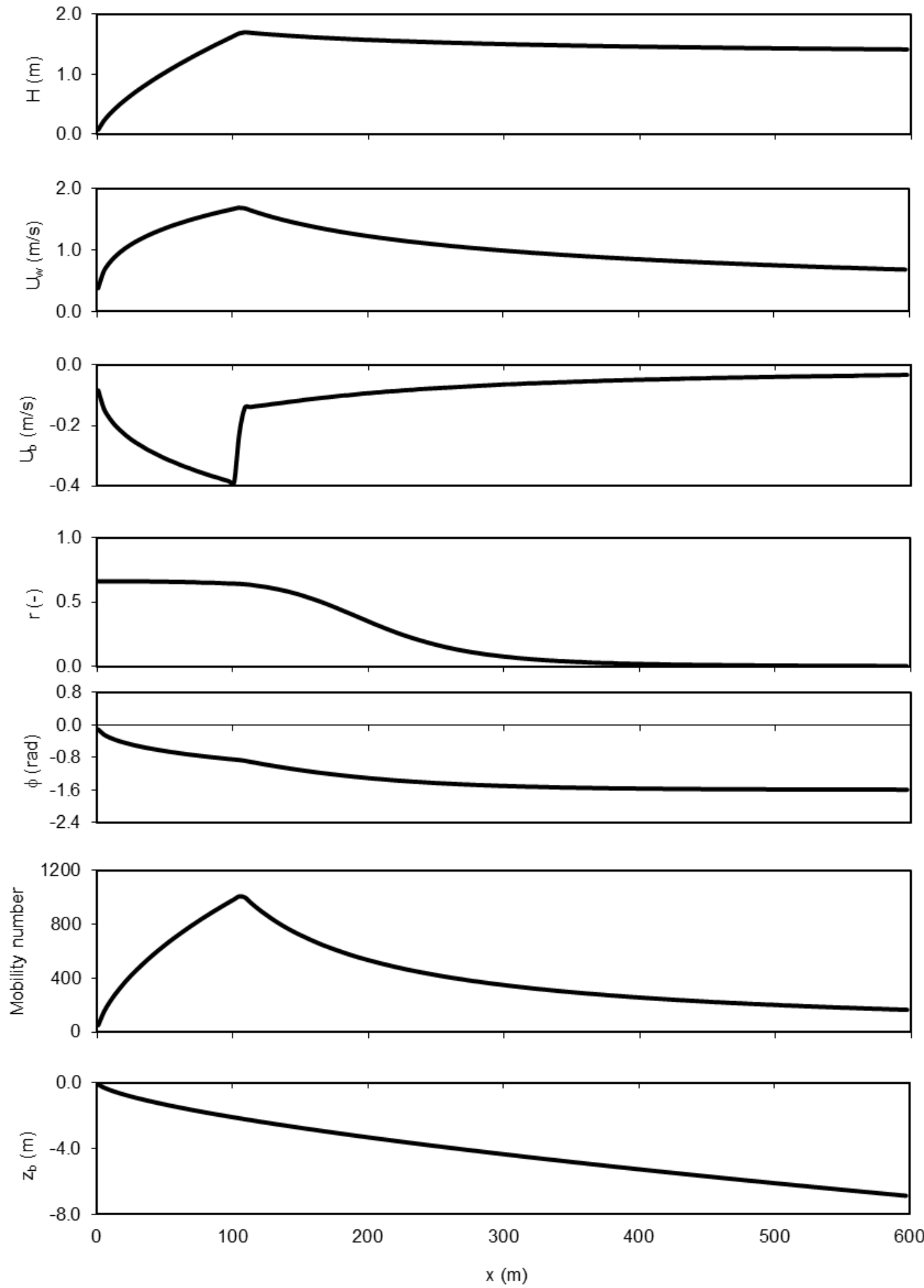

Figure 6. Cross-shore variations of $H, U_{\mathrm{w}}, U_{\mathrm{b}}, r, \phi$ and of the mobility number for a Bruun/Dean "equilibrium" profile obtained with $d_{50}=0.20 \mathrm{~mm}$.

Looking to the spatial variation of $r$ and $\phi$, a good insight of the nonlinearities can be provided. At the deepest position shown $(x=600 \mathrm{~m}), r$ is practically zero which is representative of a sinusoidal motion. However, further onshore, the value of $r$ arises almost to 0.7, evidencing that the nonlinearities are increasing. During the cross-shore variation of $r$ it is seen that $\phi$ changes from $\phi \approx-\pi / 2$ (i.e., preponderance of short, high crests) to almost zero close to the shore line. This means that, for the 
onshore positions, the wave is pitching forward (sawtooth shape) and the acceleration skewness is, now, playing an important role. Therefore, it is shown that the selected parameterizations capture efficiently the observed wave transformations from velocity-skewed in the shoaling zone, to acceleration-skewed in the inner surf and swash zone as seen in laboratory experiments (e.g., Abreu et al., 2011b; Sancho et al., 2011) and natural beaches (e.g., Elfrink et al., 2006; Ruessink et al., 2009).

Fig. 7 shows the morphological changes computed after $24 \mathrm{~h}$ of simulation, with $\Delta x=4 \mathrm{~m}$ and $\Delta t=60 \mathrm{~s}$. Two solutions referred to as A11 and N06 are presented, reflecting the consideration of the new bed shear stress predictor with $k_{\mathrm{s}}=15 d_{50}$ and $\varphi=51^{\circ}$, and Nielsen's original predictor (i.e., $S=0$ ) with $k_{\mathrm{s}}=2.5 d_{50}$ and $\varphi=51^{\circ}$, respectively. The vertical line in Fig. 7 corresponds to the initial breaking position $\left(x_{\mathrm{b}}=107.0 \mathrm{~m}\right)$. It is seen that both approaches lead to the formation of a sand bar seaward of the breaking point, resulting in the convergence of the sediment transport near the breaker line due to the skewed/asymmetric oscillatory flow of short waves and undertow current. After the formation of the bar, the waves begin to break further offshore, resulting in either the growth or the offshore migration of the bar. However, its evolution is different regarding both approaches. For N06, one registers a faster offshore migration of the bar, whereas, for A11, one assists to a larger growth of the bar. Both results exhibit erosion shoreward of the bar, which is more pronounced in the A11 solution.

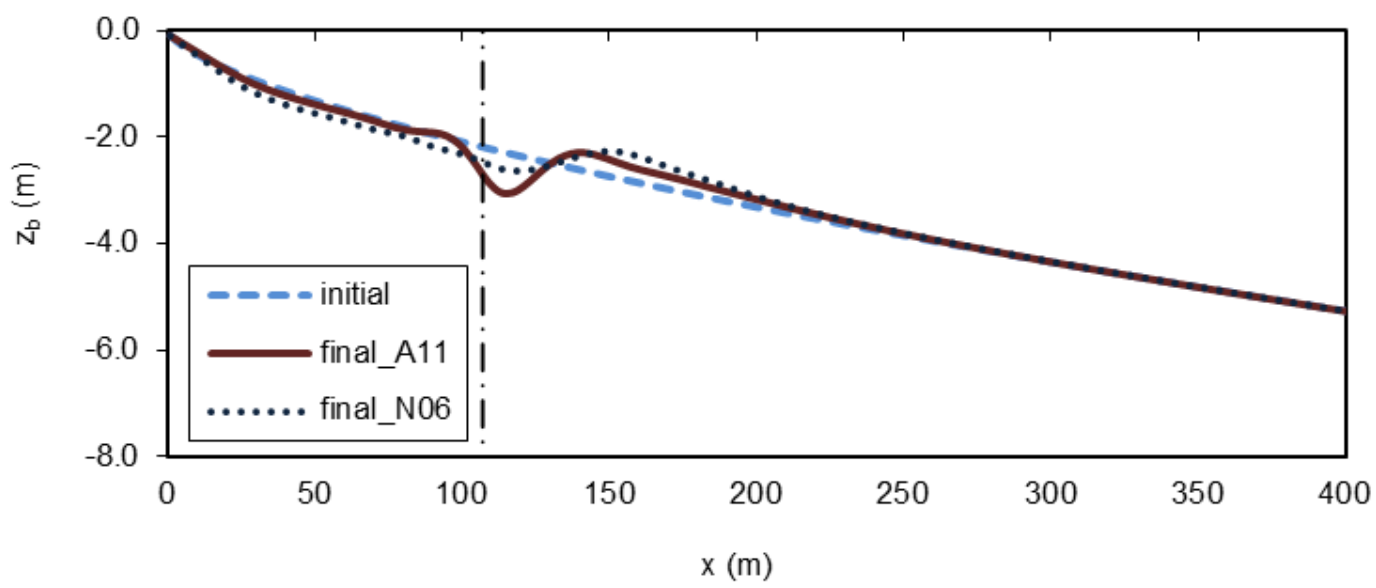

Figure 7. Initial (dashed) and final profiles of $24 \mathrm{~h}$ simulation. The final profiles consider the new bed shear stress predictor with $k_{\mathrm{s}}=15 d_{50}$ and $\varphi=51^{\circ}$ (solid) and Nielsen's original predictor (i.e., $S=0$ ) with $k_{\mathrm{s}}=2.5 d_{50}$ and $\varphi=51^{\circ}$ (dotted).

The differences in the results in Fig. 7 can be attributed to the different bed roughness values that provide different magnitudes of the wave friction factor and to the inclusion of the new term $S(t, \phi, r)$ in the new bed shear stress predictor. To illustrate it, Fig. 8 plots the position of the bar crest during the $24 \mathrm{~h}$ simulation period, considering $k_{\mathrm{s}}=2.5 d_{50}$ and $15 d_{50}$. The results show that the formation of the bar appears nearly beneath the initial breakpoint, i.e., at $x_{\mathrm{b}}=107.0 \mathrm{~m}$, due to the convergence of sediment transport at the breaker line. The consideration of $k_{\mathrm{s}}=15 d_{50}$ enhances a faster migration of the bar offshore when compared to the results with $k_{\mathrm{s}}=2.5 d_{50}$. However, it is seen that regardless of which $k_{\mathrm{s}}$ is being used, Nielsen's method (i.e., $S=0$ ) always lead to a faster offshore movement of the bar. Therefore, the inclusion of the term $S(t, \phi, r)$ is noticeably affecting the results, reducing the bar migration rate and contributing for its growth. Further validation will be pursued against field or laboratory data. 


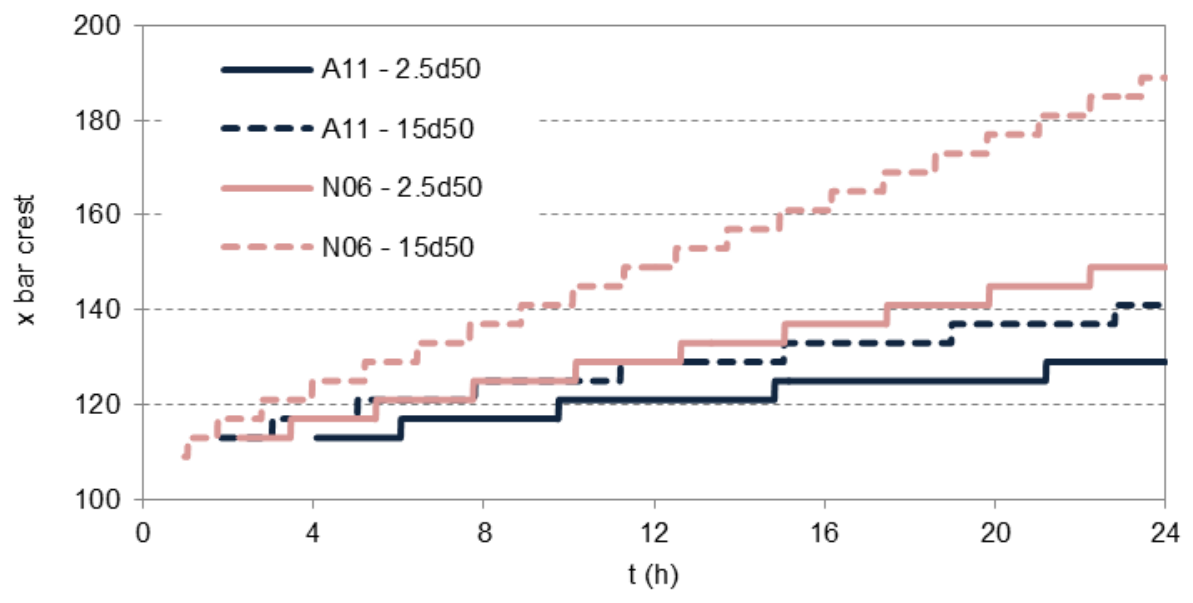

Figure 8. Position of the bar crest during the $24 \mathrm{~h}$ simulation period. The final profiles were obtained using

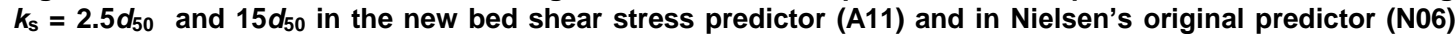
with $\varphi=51^{\circ}$.

\section{CONCLUSIONS}

This work provides further insights in the prediction of sediment transport fluxes and sandbar developments, due to the combined influence of non-linear waves with undertow currents. For that purpose, some assumptions concerning the nearshore hydrodynamics processes and sediment transport computations were made in order to be introduced in a simple numerical model capable to predict morphological changes.

A simplified wave model that predicts the wave height at each location along the cross-shore direction is presented by including two processes, namely, the wave shoaling and breaking. The parameterizations proposed by Ruessink et al. (2012) enable to characterize the orbital motions under waves with skewness and asymmetry as function of the significant wave height, wave period and water depth. The expressions provided for the two parameters associated to different degrees of the velocity and acceleration skewness, namely, $r$ and $\phi$, enable the practical application of the free-stream velocity description suggested by Abreu et al. (2010). Moreover, these parameters are also included in the new bed shear stress predictor of Abreu et al. (2011), which leads to good estimates of sediment net transport rates when inserted in the quasi-steady bed load formula of Nielsen (2006). The sediment transport predictions are assessed using the data sets of van der A et al. (2010) and Silva et al. (2011). The hydrodynamic conditions are representative of sheet flow conditions and consider different uniform grain sizes. It is shown that the results provide good estimates of the net transport rates if the bed roughness accounts for the sediment grain size.

Moreover, the sediment transport model predicts a balance between the near-bottom offshore directed sediment transport due to the undertow and the onshore transport driven by wave nonlinearities. These results corroborate the experimental trends of Silva et al. (2011), revealing that the transport model seems to reasonably capture such processes.

Furthermore, in the present work, the ability of the sediment transport model is assessed to predict beach profile evolutions by solving the bed-evolution equation. The initial bathymetry considers a Bruun/Dean "equilibrium" profile with $d_{50}=0.20 \mathrm{~mm}$, and the beach profiles computed after a $24 \mathrm{~h}$ simulation period are analysed. The cross-shore variations of $H, U_{\mathrm{w}}, U_{\mathrm{b}}, r$ and $\phi$ of the initial bathymetry are presented, showing that the selected parameterizations capture efficiently the observed wave transformations from velocity-skewed in the shoaling zone, to acceleration-skewed in the inner surf. The morphological changes are also compared with Nielsen's sediment transport model. This study shows that both approaches lead to the formation of a sand bar but differences regarding the migration and growth of the bar are noticed. Nielsen's method leads to a faster offshore migration of the bar. These differences can be mainly explained by the inclusion of the term $\mathrm{S}(t, \phi, r)$ in Abreu et al.'s (2011) bed shear stress predictor that affects the computations of sediment transport rates. 
Future work is aimed at exploring the results of the new model with different hydrodynamic conditions and its validation against observed beach profile evolutions. This will enable to show the relative strength of mechanisms associated with the wave and current induced sand transports, and the ability and weaknesses of the present (empirical) practical transport model when compared with other models.

\section{ACKNOWLEDGMENTS}

Part of this work is funded by the Research Projects PTDC/CTE-GIX/111230/2009 (EROS) and PTDC/ECM/103801/2008 (3D-MOWADI), supported by the Portuguese Foundation for Science and Technology (FCT).

\section{REFERENCES}

Abreu, T. 2011. Coastal sediment dynamics under asymmetric waves and currents: measurements and simulations. PhD thesis, University of Coimbra, $255 \mathrm{pp}$.

Abreu, T., Silva, P.A., Sancho, F. and A. Temperville. 2010. Analytical approximate wave form for asymmetric waves, Coastal Engineering, 57, 656-667.

Abreu, T., van der A, D.A., Silva, P.A., Sancho, F. and H. Michallet. 2011a. New bed shear stress estimator for net sand transport rate predictions under non-linear waves, Journal of Coastal Research, SI 64, 2007-2011.

Abreu, T., Silva, P.A. and F. Sancho. 2011b. Asymmetrical waves in barred beaches, Revista de Gestão Costeira Integrada / Journal of Integrated Coastal Zone Management, 11(3), 297-306, (in Portuguese).

Airy, G.B. 1841. Tides and waves, Encyclopaedia Metropolitana, 396 pp.

Bruun, P. 1954, Coast erosion and the development of beach profiles, Beach erosion board technical memorandum, 44, U.S. Army Engineer Waterways Experiment Station, Vicksburg, MS.

Davies, A.G., van Rijn, L.C., Damgaard, J.S., van de Graaff, J. and J.S. Ribberink. 2002. Intercomparison of research and practical sand transport models, Coastal Engineering, 46, 1-23

De Vriend, H.J., J. Zyserman, J. Nicholson, J.A. Roelvink, P. Pechon, and H.N. Southgate. 1993. Medium-term 2DH coastal area modeling, Coastal Engineering, 21, 193-224.

Dean, R.G. and R.A. Dalrymple. 1991. Water wave mechanics for engineers and scientists, Advanced Series on Ocean Engineering, 2. World Scientific, 353 pp.

Dibajnia, M., Moriya, T. and A. Watanabe. 2001. A representative wave model for estimation of nearshore local transport rate, Coastal Engineering Journal, 43(1), 1-38.

Doering, J.C. and A.J. Bowen. 1995. Parametrization of orbital velocity asymmetries of shoaling and breaking waves using bispectral analysis, Coastal Engineering, 26(1-2), 15-33.

Drake, T.G. and J. Calantoni. 2001. Discrete particle model for sheet flow sediment transport in the nearshore, Journal of Geophysical Research,106, C9, 19859-19868.

Elfrink, B., Hanes, D.M. and B.G. Ruessink. 2006. Parameterization and simulation of near bed orbital velocities under irregular waves in shallow water, Coastal Engineering, 53, 915-927.

Elgar, S.L. and R.T. Guza. 1985. Observations of bispectra of shoaling surface gravity waves, Journal of Fluid Mechanics, 167, 425-448.

Hoefel, F. and S. Elgar. 2003. Wave-induced sediment transport and sandbar migration, Science 299, 1885-1887.

Long, W., J.T. Kirby and Z. Shao, 2008. A numerical scheme for morphological bed level calculations, Coastal Engineering, 55, 167-180.

Malarkey, J. and A.G. Davies. 2012. Free-stream velocity descriptions under waves with skewness and asymmetry, Coastal Engineering, 68, 78-95.

McCowan, J. 1984. On the highest wave of permanent type, Philosophical Magazine, 38, pp. 351-358.

Meyer-Peter, E. and R. Müller. 1948. Formulas for bed-load transport. IAHR, 2nd Meeting of the International Association for Hydraulic Structures Research, Stockholm, Sweden, 39-64.

Nielsen, P. 1992. Coastal Bottom Boundary Layers and Sediment Transport, Advanced Series on Ocean Engineering, 4, World Scientific, 324 pp.

Nielsen, P. 2002. Shear stress and sediment transport calculations for swash zone modelling, Coastal Engineering, 45(1), 53-60.

Nielsen, P. 2006. Sheet flow sediment transport under waves with acceleration skewness and boundary layer streaming, Coastal Engineering, 53(9), 749-758. 
Nielsen, P. and D.P. Callaghan. 2003. Shear stress and sediment transport calculations for sheet flow under waves, Coastal Engineering, 47(3), 347-354.

O'Donoghue, T., Doucette, J.S., van der Werf, J.J. and Ribberink, J.S., 2006. The dimensions of sand ripples in full-scale oscillatory flows, Coastal Engineering, 53(12), 997-1012.

Rattanapitikon, W. and T. Shibayama. 2000. Simple model for undertow profile, Coastal Engineering Journal, 42(1), 1-30.

Richtmyer, R.D., 1962. A survey of difference methods for nonsteady fluid dynamics, Natl. Cent. Atmos. Res. Tech., Note 63-2.

Ruessink, B.G., van den Berg, T.J.J. and L.C. van Rijn. 2009. Modeling sediment transport beneath skewed asymmetric waves above a plane bed, Journal of Geophysical Research, 114(C11), C11021.

Ruessink, B.G., Ramaekers, G. and L.C. van Rijn. 2012. On the parameterization of the free-stream non-linear wave orbital motion in nearshore morphodynamic models, Coastal Engineering, 65, 5663.

Sancho, F., Abreu, T., D’Alessandro, F., Tomasicchio, G.R. and P.A. Silva. 2011. Surf hydrodynamics in front of collapsing coastal dunes. Journal of Coastal Research, SI 64: 144-148.

Shapiro, R. 1975. Linear filtering. Mathematics of computation, 29 (132), 1094-1097.

Silva, P.A., Temperville, A. and F. Seabra Santos. 2006. Sand transport under combined current and wave conditions: A semi-unsteady, practical model. Coastal Engineering, 53(11), pp. 897-913.

Silva, P.A., Abreu, T., van der A, D.A., Sancho, F., Ruessink, B.G., van der Werf, J.J. and J.S. Ribberink. 2011. Sediment transport in non-linear skewed oscillatory flows: Transkew experiments. Journal of Hydraulic Research, 49, sup1 : 72-80.

Stokes, G. G. 1847. On the theory of oscillatory waves, Trans. Cambridge Philos. Soc., 8, 441-455.

Svendsen, I.A., Madsen, P.A. and J.B. Hansen. 1978. Wave characteristics in the surf zone, 16th International Conference on Coastal Engineering, 520-539.

Thornton, E.B., Humiston, R.T. and W. Birkemeier. 1996. Bar/trough generation on a natural beach, Journal of Geophysical Research, 101(C5), 12097-12110.

Terrile, E., Reniers, A.J.H.M. and M.J.F. Stive. 2009. Acceleration and Skewness Effects on the Instantaneous Bed-Shear Stresses in Shoaling Waves, Journal of Waterway, Port, Coastal, and Ocean Engineering, 135(5), 228-234.

van der A, D.A., O'Donoghue, T. and J.S. Ribberink. 2010. Measurements of sheet flow transport in acceleration-skewed oscillatory flow and comparison with practical formulations, Coastal Engineering, 57(3), 331-342. 